\title{
Artesunate ameliorates lung fibrosis via inhibiting the Notch signaling pathway
}

\author{
YUJUAN LIU*, GUOJIN HUANG*, BIWEN MO* and CHANGMING WANG \\ Division of Respiratory Diseases, Affiliated Hospital of Guilin Medical University, Guilin, Guangxi 541001, P.R. China
}

Received June 30, 2016; Accepted March 31, 2017

DOI: $10.3892 / e t m .2017 .4573$

\begin{abstract}
The present study aimed to determine the underlying molecular mechanism of the antifibrotic effect of artesunate in pulmonary fibrosis (PF). Primary lung fibroblasts were isolated from the lung tissues of rats, and treated with artesunate $(8 \mu \mathrm{g} / \mathrm{ml})$ and transforming growth factor (TGF)- $\beta 1(5 \mathrm{ng} / \mathrm{ml})$. For in vivo experiments, the rats were administered bleomycin intratracheally, followed by daily intraperitoneal artesunate injections for 27 days. Western blotting, and immunohistochemical and immunofluorescent staining were used to assess the expression of key components of the Notch signaling pathway, including $\alpha$-smooth muscle actin ( $\alpha$-SMA) and type IV collagen. Artesunate $(8 \mu \mathrm{g} / \mathrm{ml})$ was identified to inhibit TGF- $\beta 1$-induced $\alpha$-SMA and collagen protein expression, and repress the Notch signaling pathway, in primary lung fibroblasts. Downregulation of $\alpha$-SMA and collagen by artesunate was associated with inhibition of the Notch signaling pathway. The daily intraperitoneal injection of artesunate $(1 \mathrm{mg} / \mathrm{kg})$ in rats was determined to inhibit bleomycin-induced overexpression of $\alpha$-SMA and type IV collagen proteins, and inhibit the Notch signaling pathway, in lung tissues. In conclusion, the results of the current study indicate that artesunate inhibits the TGF- $\beta 1$-induced differentiation of rat primary lung fibroblasts into myofibroblasts and ameliorates bleomycin-induced PF. In addition, the results of the present study suggest that the underlying molecular mechanism for these effects of artesunate is repression of the Notch signaling pathway.
\end{abstract}

\section{Introduction}

Idiopathic pulmonary fibrosis (IPF) is a progressive, severely debilitating disease with a high mortality rate (1). However,

Correspondence to: Professor Changming Wang, Division of Respiratory Diseases, Affiliated Hospital of Guilin Medical University, 15 Lequn Road, Guilin, Guangxi 541001, P.R. China E-mail: 1507959392@qq.com

*Contributed equally

Key words: artesunate, lung fibrosis, notch signaling pathway there are currently no effective treatments for IPF. The clinical features of IPF include chronic ( $>6$ months) exertional shortness of breath, a dry cough, inspiratory bibasilar crackles and finger clubbing $(2,3)$. Although the risk factors for IPF remain unclear, a prevailing hypothesis suggests that injuries in the alveolar epithelium trigger pathophysiological alterations, including increased production of transforming growth factor (TGF)- $\beta$, which induces the differentiation of fibroblasts into myofibroblasts and the excessive deposition of extracellular matrix (ECM) (4). The alteration of fibroblasts and the ECM serves a major role in the development of fibrosis, and creates a profibrosis positive feedback loop (5). The differentiation of fibroblasts into myofibroblasts, which are fibroblasts that contain $\alpha$-smooth muscle actin ( $\alpha$-SMA) and other contractile elements, has been identified as a key event in the development of IPF and other profibrotic conditions $(6,7)$.

The neurogenic locus notch homolog protein (Notch) signaling pathway is highly conserved and serves a key role in cellular proliferation, specification and differentiation (8). In mammals, there are four types of Notch receptor (Notch1-4) and five Notch receptor ligands (Jagged1, Jagged2, delta-like 1, delta-like 3 and delta-like 4). Notch receptors are activated by ligand binding, upon which the Notch intracellular domain (NICD) is released and translocates into the nucleus, where it interacts with transcriptional repressors to modulate the expression of target genes that include the well-characterized transcription factor hairy enhance of split (Hes).

Previous studies have reported that the Notch signaling pathway is associated with human fibrotic diseases, including pulmonary fibrosis (PF) $(9,10)$. Artesunate, the recommended first-line treatment for severe malarias (11), was recently demonstrated by our group to inhibit the proliferation of lung fibroblast by promoting apoptosis and reducing collagen secretion (12). In addition, artesunate was determined to downregulate the expression of TGF- $\beta 1$, mothers against decapentaplegic homolog 3 , heat shock protein $47, \alpha$-SMA and collagen type I (12).

The present study aimed to evaluate the effect of artesunate on the TGF- $\beta 1$-induced differentiation of lung fibroblasts into myofibroblast. In addition, the effect of artesunate on bleomycin-induced pulmonary fibrosis in rats was investigated. Furthermore, the potential underlying molecular mechanisms of the effects of artesunate on these processes was explored. 


\section{Materials and methods}

Materials. Antibodiesfor cleaved Notch(NICD,cat.no.ab52301), $\alpha$-SMA (cat. no. ab5694) and Hes-1 (cat. no. ab108937) were purchased from Abcam (Cambridge, MA, USA). Antibodies for Jagged1 (cat. no. Sc-8303) were obtained from Santa Cruz Biotechnology, Inc. (Dallas, TX, USA). Antibodies for Notch1 (cat. no. 4380) were purchased from Cell Signaling Technology, Inc. (Danvers, MA, USA). Antibodies for $\beta$-actin (cat. no. PR0255), horseradish peroxidase (HRP)-conjugated anti-mouse or -rabbit immunoglobulin $\mathrm{G}$ antibodies (cat. no ZB-2301 and ZB-2305), and Fluorescein isothiocyanate (FITC)-conjugated anti-rabbit immunoglobulin G antibody (cat. no. ZF-0311) were obtained from Beijing Zhongshan Golden Bridge Biotechnology Co., Ltd. (Beijing, China). The $\gamma$-secretase inhibitor DAPT was purchased from Sigma-Aldrich (Merck KGaA, Darmstadt, Germany). Human recombinant TGF- $\beta 1$ was purchased from Peprotech, Inc. (Rocky Hill, NJ, USA). Penicillin/streptomycin, fetal bovine serum (FBS) and Dulbecco's modified Eagle's medium (DMEM) were obtained from Invitrogen (Thermo Fisher Scientific, Inc., Waltham, MA, USA). Bleomycin hydrochloride for injection was purchased from Nippon Kayaku Co., Ltd. (Tokyo, Japan). Artesunate was purchased from Guilin Pharmaceutical (Shanghai) Co., Ltd. (Guilin, China). The DAB kit (cat. no. ZLI-9017) was obtained from Beijing Zhongshan Golden Bridge Biotechnology Co., Ltd. (Beijing, China) according to the manufacturer's protocol. Other reagents were purchased from Sigma-Aldrich (Merck KGaA).

Lung fibroblast culture. Lung fibroblasts were isolated from the rats described below according to a previous method (13). The cells were cultured $\left(\right.$ at $37^{\circ} \mathrm{C}, 95 \%$ humidity and $5 \% \mathrm{CO}_{2}$ ) in DMEM supplemented with $10 \%$ FBS. The identity of fibroblast cells was verified at passage four by testing the expression of vimentin (1:200 dilution; cat. no. 5741; CST, Inc., Danvers, MA, USA) by immunofluorescence staining. Cells at passage 5 were used for further experiments.

The primary fibroblasts were synchronized by incubation (at $37^{\circ} \mathrm{C}$ in $5 \% \mathrm{CO}_{2}$ ) in serum-free DMEM for $24 \mathrm{~h}$ and then randomly divided into five groups as follows: The control group, incubated in serum-free DMEM; the TGF- $\beta 1$ group, incubated in DMEM containing $5 \mathrm{ng} / \mathrm{ml}$ TGF- $\beta 1$; the Notch inhibitor $(\mathrm{DAPT})+$ TGF- $\beta 1$ group, incubated in DMEM containing $5 \mathrm{ng} / \mathrm{ml}$ TGF- $\beta 1$ and $10 \mu \mathrm{M}$ DAPT; the artesunate + TGF- $\beta 1$ group, incubated in DMEM containing $5 \mathrm{ng} / \mathrm{ml} \mathrm{TGF-} \beta 1$ and $8 \mu \mathrm{g} / \mathrm{ml}$ artesunate; and the artesunate control group, incubated in DMEM containing $8 \mu \mathrm{g} / \mathrm{ml}$ artesunate at $37^{\circ} \mathrm{C}$ for $24 \mathrm{~h}$.

Animal experiment protocols. Male Sprague Dawley rats $(\mathrm{n}=24)$ weighing 180-250 $\mathrm{g}$ at 8 weeks old were obtained from the Center for Experimental Animals at Guilin Medical University (Guilin, China). The rats were housed in specific pathogen-free conditions at $20-24^{\circ} \mathrm{C}$ and humidity $30-50 \%$ with a 12 -h light/dark cycle and ad libitum access food and water. The animal protocol was reviewed and approved by the Institutional Animal Care and Use Committee of the Affiliated Hospital of Guilin Medical University (Guilin, China).

For in vivo study, the rats were randomly divided into the following four groups: The control group $(n=6)$, which received intratracheal administration of $0.9 \% \mathrm{NaCl}$ solution on day
1, followed by daily intraperitoneal injections of $0.9 \% \mathrm{NaCl}$ solution $(1 \mathrm{ml})$ for 27 days; the bleomycin group $(\mathrm{n}=6)$, which received intratracheal administration of bleomycin $(5 \mathrm{mg} / \mathrm{kg})$ at day 1 , followed by daily intraperitoneal injections of $0.9 \% \mathrm{NaCl}$ solution $(1 \mathrm{ml})$ for 27 days; the artesunate group $(\mathrm{n}=6)$, which received intratracheal administration of $0.9 \% \mathrm{NaCl}$ solution at day 1 , followed by daily intraperitoneal injections of artesunate $(100 \mathrm{mg} / \mathrm{kg})$ for 27 days; and the bleomycin + artesunate group $(\mathrm{n}=6)$, which received intratracheal administration of bleomycin $(5 \mathrm{mg} / \mathrm{kg})$ at day 1 , followed by daily intraperitoneal injection of artesunate $(100 \mathrm{mg} / \mathrm{kg})$ for 27 days. All animals were euthanized by pentobarbital overdose $(100 \mathrm{mg} / \mathrm{kg})$ at the end of treatment period, and lung tissues were quickly removed and processed for further analysis.

Masson's trichrome staining. Masson's trichrome staining was performed to observe lung fibroblast collagen secretion. Lung fibroblasts $\left(0.3 \times 10^{6} /\right.$ well $)$ were plated in 6 -well plates with or without TGF- $\beta 1(5 \mathrm{ng} / \mathrm{ml})$ at $37^{\circ} \mathrm{C}$ in $\left.5 \% \mathrm{CO}_{2}\right)$ for $24 \mathrm{~h}$, and the cultured cells were washed 3 times for 1 min each with ice-cold PBS and fixed at $4^{\circ} \mathrm{C}$ with $4 \%$ paraformaldehyde for $30 \mathrm{~min}$. Then lung fibroblasts were stained with Masson trichrome staining kit manual at $20-24^{\circ} \mathrm{C}$ for $5 \mathrm{~min}$ and subsequently examined with a light microscope (Olympus Corporation, Beijing, China).

Immunofluorescence staining. Lung fibroblasts $(7,000$ cells per well) were plated on 6-well chamber slides with or without TGF- $\beta 1(5 \mathrm{ng} / \mathrm{ml})$ at $37^{\circ} \mathrm{C}$ for $24 \mathrm{~h}$, then the culture medium was then aspirated, and the slides were washed with PBS and fixed with $4 \%$ paraformaldehyde at room temperature for $20 \mathrm{~min}$. Cells were permeabilized with $0.05 \%$ Triton $\mathrm{X}-100$ at $4^{\circ} \mathrm{C}$ for 10 min and blocked with $3 \%$ bovine serum albumin (BSA; cat. no. OR0015; Leagene Co., Ltd. Beijing, China) for at $4^{\circ} \mathrm{C} 1 \mathrm{~h}$. The slides were incubated at $4^{\circ} \mathrm{C}$ overnight with primary antibodies directed against $\alpha$-SMA (dilution, 1:100 in 3\% BSA) or against vimentin (1:200 dilution; cat. no. 5741; CST, Inc.). Subsequently, the slides were washed with PBS and incubated with secondary anti-rabbit Texas green-conjugated antibodies at $4^{\circ} \mathrm{C}$ (dilution, 1:200 in 3\% BSA). Slides were then washed in PBS for 20 min. Two drops (10-15 $\mu$ l) of ProLong ${ }^{\circledR}$ Gold Antifade Mountant (Thermo Fisher Scientific, Inc.) was added to each well prior to the addition of coverslips. The slides were then examined by fluorescence microscopy.

Western blot analysis. The lung tissue was homogenized in radioimmunoprecipitation assay (RIPA) buffer with a protease inhibitor cocktail (cat. no. 04693159001; Roche Diagnostics, Basel, Switzerland) containing $1 \mathrm{mmol} / \mathrm{l}$ phenylmethanesulfonyl fluoride (PMSF) using a tissue grinder. Cultured cells were lysed on ice in ice-cold RIPA buffer with a protease inhibitor cocktail containing $1 \mathrm{mmol} / \mathrm{l}$ PMSF for $20 \mathrm{~min}$. Proteins from lung tissue and fibroblast were prepared as previously described $(12,13)$. A total of $20 \mu \mathrm{g}$ protein lane was loaded into each lane and separated via 10\% SDS-PAGE gel and then transferred to a polyvinylidene difluoride membrane, which was blocked with 5\% non-fat milk in Tris-buffered saline (TBS) at room temperature for $1.5 \mathrm{~h}$. The membranes were then incubated at $4^{\circ} \mathrm{C}$ overnight with cleaved Notch (NICD 1:1,000 dilution; cat. no. ab52301), $\alpha$-SMA (1:1,000 dilution; 
A

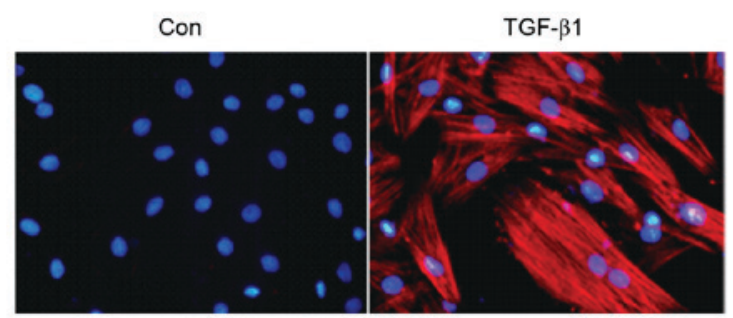

C

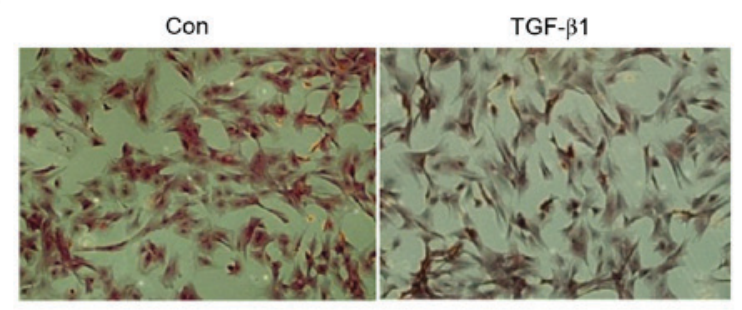

B
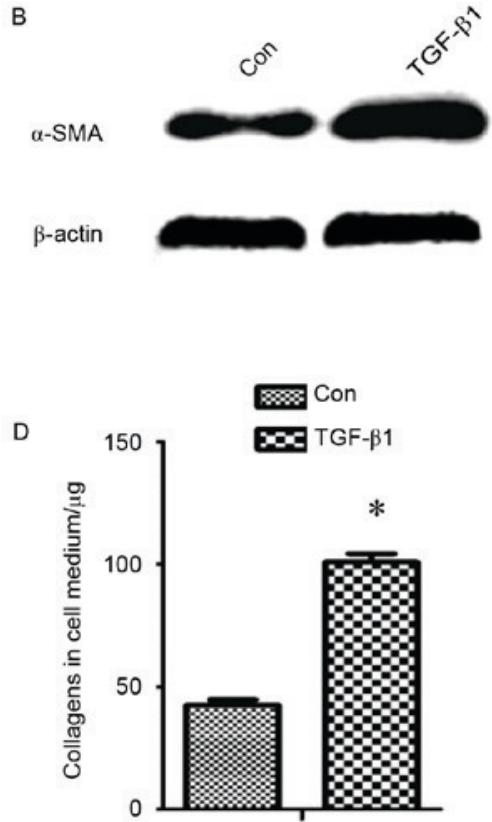

Figure 1 . TGF- $\beta 1$ induces the expression of $\alpha$-SMA and collagens in primary lung fibroblasts. Primary lung fibroblasts were treated with TGF- $\beta 1$ for 24 h, then the expression of $\alpha$-SMA was detected by (A) immunofluorescence (magnification, x200) and (B) western blotting. (C) Primary lung fibroblasts were treated with TGF- $\beta 1$ for $24 \mathrm{~h}$ and collagens were detected by Masson's trichrome staining (magnification, $\mathrm{x} 200$ ). (D) Primary lung fibroblasts were treated with TGF- $\beta 1$ for $24 \mathrm{~h}$; the collagens in cell medium were measured using the Sirius Red Collagen Detection kit. Con, control group; TGF- $\beta 1$, transforming growth factor $\beta 1 ; \alpha$-SMA, $\alpha$ smooth muscle actin. ${ }^{*} \mathrm{P}<0.05$ vs. the Con group.

cat. no. ab5694), Hes-1 (1:800 dilution; cat. no. ab108937), Jagged1 (1:500 dilution; cat. no. Sc-8303) and Notch1 (1:400 dilution; cat. no. 4380), followed by three washes with TBS containing Tween-20 (5 min/wash). The membrane was subsequently incubated with secondary antibodies, horseradish peroxidase-conjugated anti-mouse or anti-rabbit immunoglobulin $\mathrm{G}$ antibodies (1:5,000 dilution; Beijing Zhongshan Golden Bridge Biotechnology Co., Ltd.), cat. no ZB-2301 and ZB-2305, respectively) at $20-24^{\circ} \mathrm{C}$ for $1.5 \mathrm{~h}$. Protein bands were detected using Enhanced Chemiluminescent reagent and imaged with a ChemiDoc MP Imaging system (Bio-Rad Laboratories, Inc., Hercules, CA, USA).

Immunohistochemical staining. Tissues were fixed with formalin at $20-24^{\circ} \mathrm{C}$ for $24 \mathrm{~h}$ and embedded with paraffin, then sliced into $5 \mu \mathrm{m}$ thick sections, which were deparaffinized in xylene followed by rehydration in a graded series of alcohols. Antigen retrieval was performed through pressure cooking (at $121^{\circ} \mathrm{C}$ ) in citrate buffer solution for $3 \mathrm{~min}$. Slides were rinsed with PBS and then incubated in 3\% hydrogen peroxide solution for at $20-24^{\circ} \mathrm{C}$ for $20 \mathrm{~min}$. After rinsing with PBS three times, the slides were incubated with $10 \%$ fetal bovine serum (cat. no. 10437028; Gibco; Thermo Fisher Scientific, Inc.) in PBS for 30 min to block nonspecific binding. The slides were then incubated with primary antibodies directed against Jagged1 (1:400), NICD (1:200), Hes-1 (1:200), $\alpha$-SMA (1:200) or Type-IV collagen (1:400 dilution; cat. no. ab6586; Abcam) at $4^{\circ} \mathrm{C}$ overnight, followed by three washes in PBS (5 min/wash). Subsequently, the slides were incubated with biotinylated goat anti-rabbit polyclonal secondary antibody (cat. no. XIT-9901; Maixin-Bio, Fuzhou, China) at room temperature for $30 \mathrm{~min}$, followed by three washes in PBS ( $5 \mathrm{~min} / \mathrm{wash})$. The slides were then stained with the DAB kit at $20-24^{\circ} \mathrm{C}$ for $5-10$ min according to manufacturer's instruction. Finally, the slides were dehydrated, cleared with xylene and mounted with neutral gum. Images of tissue sections were captured using a BX53 digital light microscope (Olympus Corporation, Tokyo, Japan).

ECM-deposition assay. Lung fibroblasts (0.3×10\% $/$ well in 6-well plate) were stimulated by incubation with TGF- $\beta 1(5 \mathrm{ng} / \mathrm{ml})$ for $24 \mathrm{~h}$ at $37^{\circ} \mathrm{C}$ with or without artesunate $(8 \mu \mathrm{g} / \mathrm{ml})$ or DAPT $(10 \mu \mathrm{mol} / 1)$, then in serum-free DMEM at $37^{\circ} \mathrm{C}$ for $48 \mathrm{~h}$. Collagen secretion and deposition (collagen type I-V) were determined using the Sirius Red Collagen Detection kit (cat. no. 9062; Chonrex, Inc., Redmond, WA, USA) according to the manufacturer's protocol.

Statistical analysis. Statistical tests were performed using SPSS software (version 18.0; SPSS, Inc., Chicago, IL, USA). Results are presented as the mean \pm standard error. The statistical significance of differences between groups was determined using one-way analysis of the variance with a post hoc Tukey's range test. $\mathrm{P}<0.05$ was considered to indicate a statistically significant difference.

\section{Results}

TGF- $\beta 1$ induces the differentiation of rat primary lung fibroblasts. To assess whether TGF- $\beta 1$ serves a role during the differentiation of primary lung fibroblasts into myofibroblasts, the expression of $\alpha$-SMA, a myofibroblast marker and all type of collagen, was detected (Fig. 1). This revealed that protein levels of $\alpha$-SMA (Fig. 1A and B) and collagens (Fig. 1C and D) were increased in TGF- $\beta 1$-treated fibroblasts compared with the control group, indicating that TGF- $\beta 1$ induces the differentiation of fibroblast into myofibroblasts. 


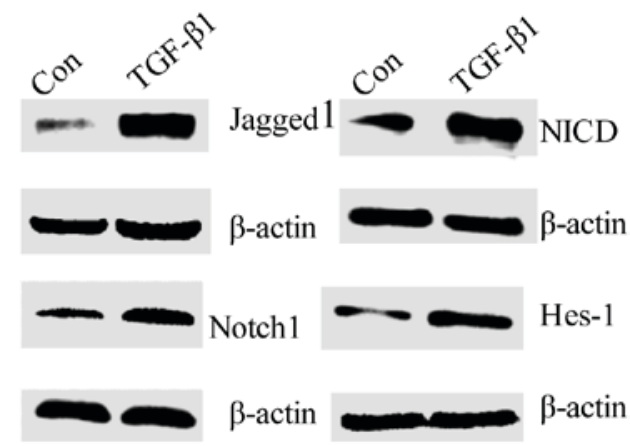

Figure 2. TGF- $\beta 1$ activates the Notch signaling pathway in primary lung fibroblasts. Representative western blotting bands are presented and $\beta$-actin was used as loading control. Con, control group; TGF- $\beta 1$, transforming growth factor $\beta 1$; Notch1, neurogenic locus notch homolog protein 1 ; NICD, Notch intracellular domain; Hes-1, hairy enhance of split 1.
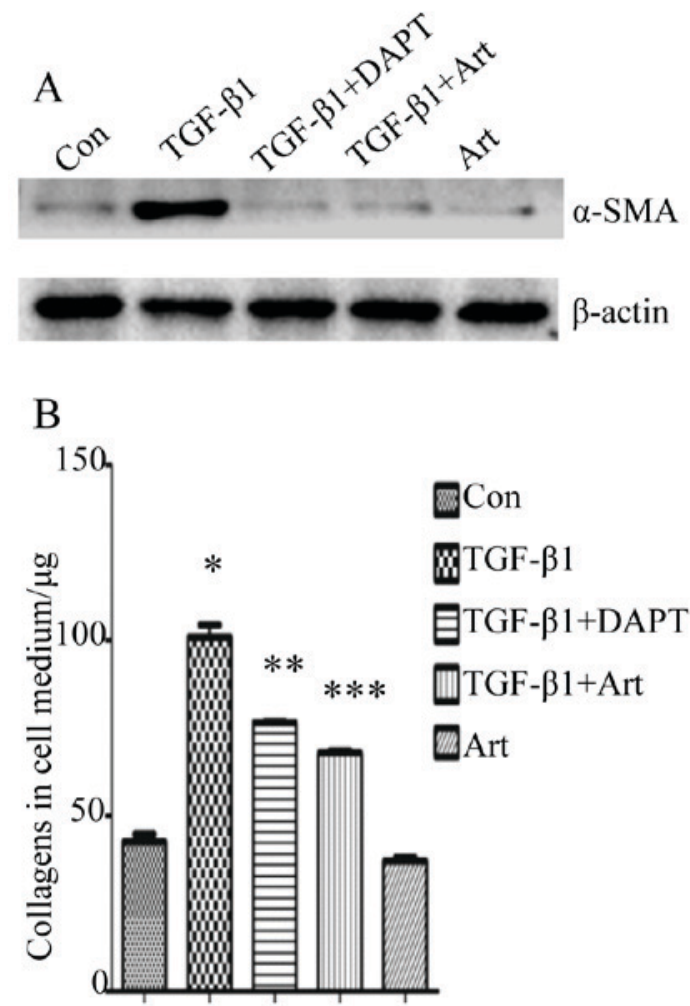

Figure 3. Artesunate inhibits the expression of $\alpha$-SMA and collagens in primary lung fibroblasts. (A) Representative western blotting results for $\alpha$-SMA protein expression in lung fibroblasts. (B) The amount of collagens in the culture medium of primary lung fibroblasts ${ }^{*} \mathrm{P}<0.05,{ }^{* * *} \mathrm{P}<0.01$ and ${ }^{* * *} \mathrm{P}<0.001$ vs. the Con group. Con, control group; TGF- $\beta 1$, transforming growth factor $\beta 1$; Art, artesunate; $\alpha$-SMA, $\alpha$ smooth muscle actin.

TGF- $\beta 1$ activates the Notch signaling pathway in primary lung fibroblasts. The Notch signaling pathway is an important signaling pathway in PF (8). To investigate the role of the Notch signaling pathway in the TGF- $\beta 1$-induced differentiation of fibroblasts into myofibroblasts, the protein expression of Jagged1, Notch1, NICD and Hes-1 was measured. This revealed that all were increased in TGF- $\beta 1$-treated fibroblasts compared with the control group (Fig. 2). Furthermore, DAPT, a $\gamma$-secretase inhibitor and inhibitor of the Notch signaling

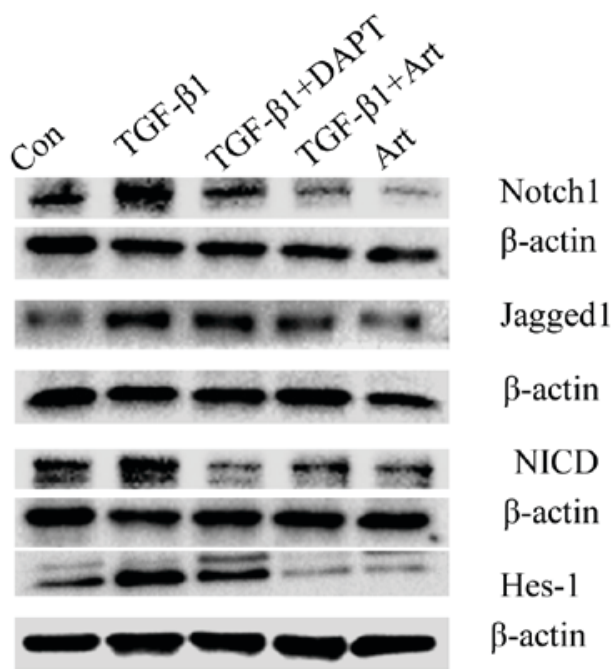

Figure 4. Artesunate inhibits the expression of components of the Notch signaling pathway in primary lung fibroblasts. Representative western blot analysis results for Notch1, Jagged1, NICD and Hes-1 protein. Con, control group; TGF- $\beta 1$, transforming growth factor $\beta 1$; Art, artesunate; $\alpha$-SMA $\alpha$ smooth muscle actin; Notch1, neurogenic locus notch homolog protein 1 ; NICD, Notch intracellular domain; Hes-1, hairy enhance of split 1.

pathway, significantly suppressed the TGF- $\beta 1$-induced overexpression of $\alpha$-SMA $(\mathrm{P}<0.01$; Fig. 3). These findings indicate that the Notch signaling pathway is associated with the TGF- $\beta 1$-induced differentiation of fibroblasts into myofibroblasts.

Artesunate inhibits the differentiation of primary lung fibroblasts. Based on our groups previous finding that artesunate exerts an antiprofibrotic effect in rats with bleomycin-induced $\mathrm{PF}$ (12), and the finding of the current study that the expression of $\alpha$-SMA and collagens was upregulated in TGF- $\beta 1$-treated fibroblasts (Fig. 1), it was hypothesized that artesunate may serve a role in the differentiation of lung fibroblasts. To test this hypothesis, the effect of artesunate on the expression of $\alpha$-SMA and collagens in TGF- $\beta 1$-treated lung fibroblasts was examined. The protein expression of $\alpha$-SMA (Fig. 3A) and the amount of collagens secreted in culture medium (Fig. 3B) were significantly decreased after treatment with artesunate. In addition, the effect of artesunate was only slightly less compared with that of DAPT (Fig. 3). These findings suggest that artesunate is able to inhibit the differentiation of fibroblasts into myofibroblasts.

Artesunate inhibits the Notch signaling pathway in primary lung fibroblasts. The aforementioned experiments indicated that the Notch signaling pathway serves a role in the TGF- $\beta 1$-induced differentiation of fibroblasts to myofibroblasts, and that artesunate is able to inhibit this. To evaluate whether the inhibitory effect of artesunate on this differentiation is via the Notch signaling pathway, the protein levels of Notch1, Jagged1, NICD and Hes-1 in lung fibroblasts treated with artesunate was measured. The expression of these proteins was markedly decreased in lung fibroblasts following treatment with Art or DAPT compared with the control group (Fig. 4), suggesting that artesunate inhibits the Notch signaling pathway. 

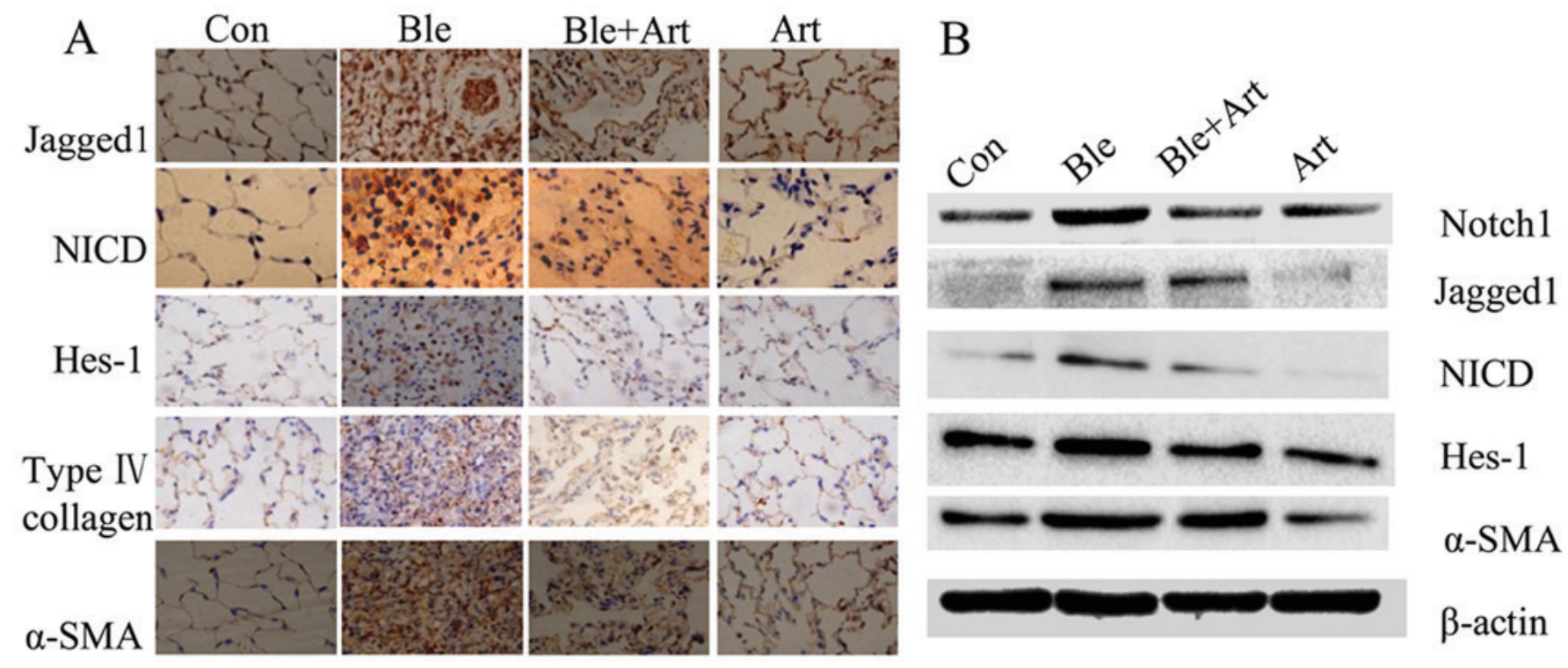

Figure 5. Artesunate inhibits the expression of collagen, $\alpha$-SMA and components of the Notch signaling pathway in the lung tissues of rats with bleomycin-induced pulmonary fibrosis. Representative (A) immunohistochemical images (magnification x40) and (B) western blotting results for Jagged1, NICD, Hes-1, collagen and $\alpha$-SMA. Con, control group; Ble, bleomycin; Art, artesunate; $\alpha$-SMA, $\alpha$ smooth muscle actin; Notch1, neurogenic locus notch homolog protein 2; NICD, Notch intracellular domain; Hes-1, hairy enhance of split 1.

Artesunate suppresses the expression of collagens, $\alpha$-SMA and certain components of the Notch signaling pathway in the lung tissues of rats with bleomycin-induced PF. Bleomycin induces inflammation and collagen deposition in the lungs of rats, and a previous study by our previously groups demonstrated that artesunate can ameliorate these pathological alterations in cultured frbroblasts (12). In the present study, an in vivo experiement was performed to confirm these findings. Immunohistochemistry (Fig. 5A) and western blotting (Fig. 5B) were employed to detect the expression of collagen, $\alpha$-SMA and certain key components of the Notch signaling pathway in the lung tissue from rats with bleomycin-induced PF treated with artesunate. The results demonstrated that the protein levels of collagen, $\alpha$-SMA, Jagged1, Notch, NICD and Hes-1 were markedly increased following exposure to bleomycin, indicating that the Notch signaling pathway is activated by bleomycin. However, the protein levels of collagen, $\alpha$-SMA, Jagged1, Notch1, NICD and Hes-1 were decreased in the lung tissues from rats treated with artesunate, or bleomycin and artesunate, suggesting that artesunate inhibits the Notch signaling pathway.

\section{Discussion}

The results of the present study indicate that artesunate inhibits the TGF- $\beta 1$-induced expression of collagen and $\alpha$-SMA in lung fibroblasts, and that artesunate reduces bleomycin-induced PF in rats. In addition, artesunate was identified to downregulate key components of the Notch signaling pathway in vitro and in vivo, including Jagged1, Notch1, NICD and Hes-1.

$\mathrm{PF}$ is a chronic lung disorder characterized by the dysregulated recruitment, proliferation and differentiation of fibroblasts, excessive deposition of ECM and abnormal lung remodeling (4). Fibroblasts serve an important role in the pathogenesis of $\mathrm{PF}$, and several factors influence their proliferation and synthesis of ECM (14). Myofibroblasts, a marker of fibrotic diseases, secrete ECM, collagens and a-SMA, which leads to the loss of alveolar function $(4,14)$. Numerous profibrotic cytokines, including TGF- $\beta 1$, platelet-derived growth factor and tumor necrosis factor $\alpha$, serve roles in the differentiation of fibroblasts into myofibroblasts. The present study demonstrated that TGF- $\beta 1$ could induce the differentiation of fibroblasts into myofibroblasts, in addition to activating the Notch signaling pathway.

There are few reports on the role of the Notch signaling pathway in the differentiation of lung fibroblasts into myofibroblasts. The Notch signaling pathway is a highly conserved pathway, which is essential to normal embryonic development, cellular proliferation, specification and differentiation, and is associated with fibrotic disease (8). The findings of the present study aid in the better understanding of the pathophysiology of lung fibrosis, in addition to suggesting that the Notch signaling pathway is a potential therapeutic target for this disease. Since overexpression of Hes1 enhances the promoter activities of $\alpha$-SMA and collagen type $1 \alpha 2$ (15), therefore TGF- $\beta 1$ may function, at least partially, via the Notch signaling pathway to upregulate Hes1 expression and promote $\alpha$-SMA expression.

The results of the present study are consistent with previous reports that Notch deficiency has a significant inhibitory effect on the response to bleomycin-induced PF $(10,16)$, suggesting that cellular signaling pathway crosstalk serves an essential role in the pathogenesis of PF. Our group recently reported that artesunate, a drug used for the treatment of severe malaria in adults and children worldwide, may inhibit the development of PF $(12,17)$. Previous experiments by our group demonstrated artesunate can induce the apoptosis of fibroblasts, inhibit TGF- $\beta 1$-induced epithelial-to-mesenchymal transition and downregulate the expression of TGF- $\beta 1$ in an animal model of PF $(12,18)$. The present study provided additional evidence revealing the potential underlying molecular mechanism of the antifibrotic effect of artesunate. However, further experiments are required to test whether artesunate functions via the same mechanism in other types of pulmonary cells. 
In conclusion, the current study demonstrated that artesunate effectively inhibits the Notch signaling pathway and the TGF- $\beta 1$-induced differentiation of primary lung fibroblasts into myofibroblasts in vitro, and ameliorates $\mathrm{PF}$ in vivo. The results of the present study indicate that the Notch signaling pathway serves a role in the differentiation of fibroblasts into myofibroblasts, and may be a potential novel therapeutic target for PF.

\section{Acknowledgements}

The present study was supported the Natural Science Foundation of Guangxi (grant nos. 2014GXNSFAA118151 and 2015GXNSFAA139178), the National Natural Science Foundation of China (grant no. 81360010), and the Guangxi Health and Family Planning Commission (grant no. S2015-34). G. H. was supported by the Hundred Talents Program of Guangxi.

\section{References}

1. Puglisi S, Torrisi SE, Giuliano R, Vindigni V and Vancheri C: What we know about the pathogenesis of idiopathic pulmonary fibrosis. Semin Respir Crit Care Med 37: 358-367, 2016.

2. Kim HJ, Perlman D and Tomic R: Natural history of idiopathic pulmonary fibrosis. Respir Med 109: 661-670, 2015.

3. Raghu G, Collard HR, Egan JJ, Martinez FJ, Behr J, Brown KK, Colby TV, Cordier JF, Flaherty KR, Lasky JA, et al: An official ATS/ERS/JRS/ALAT statement: Idiopathic pulmonary fibrosis: Evidence-based guidelines for diagnosis and management. Am J Respir Crit Care Med 183: 788-824, 2011.

4. King TE Jr, Pardo A and Selman M: Idiopathic pulmonary fibrosis. Lancet 378: 1949-1961, 2011.

5. Parker MW, Rossi D, Peterson M, Smith K, Sikström K, White ES, Connett JE, Henke CA, Larsson O and Bitterman PB: Fibrotic extracellular matrix activates a profibrotic positive feedback loop. J Clin Invest 124: 1622-1635, 2014.
6. Weiskirchen R and Tacke F: Liver fibrosis: From pathogenesis to novel therapies. Dig Dis 34: 410-422, 2016.

7. Pardo A, Cabrera S, Maldonado M and Selman M: Role of matrix metalloproteinases in the pathogenesis of idiopathic pulmonary fibrosis. Respir Res 17: 23, 2016.

8. Artavanis-Tsakonas S, Rand MD and Lake RJ: Notch signaling: Cell fate control and signal integration in development. Science 284: 770-776, 1999.

9. Zhou Y, Liao S, Zhang Z, Wang B and Wan L: Astragalus injection attenuates bleomycin-induced pulmonary fibrosis via down-regulating Jagged1/Notch1 in lungs. J Pharm Pharmacol 68: 389-396, 2016.

10. Liu T, Hu B, Choi YY, Chung M, Ullenbruch M, Yu H, Lowe JB and Phan SH: Notch1 signaling in FIZZ1 induction of myofibroblast differentiation. Am J Pathol 174: 1745-1755, 2009.

11. Reyburn $\mathrm{H}$ : New WHO guidelines for the treatment of malaria. BMJ 340: c2637, 2010.

12. Wang C, Xuan X, Yao W, Huang G and Jin J: Anti-profibrotic effects of artesunate on bleomycin-induced pulmonary fibrosis in Sprague Dawley rats. Mol Med Rep 12: 1291-1297, 2015.

13. Wang Y, Huang G, Mo B and Wang C: Artesunate modulates expression of matrix metalloproteinases and their inhibitors as well as collagen-IV to attenuate pulmonary fibrosis in rats. Genet Mol Res 15, 2016.

14. Hardie WD, Glasser SW and Hagood JS: Emerging concepts in the pathogenesis of lung fibrosis. Am J Pathol 175: 3-16, 2009.

15. Zhang K, Zhang YQ, Ai WB, Hu QT, Zhang QJ, Wan LY, Wang XL, Liu CB and Wu JF: Hes1, an important gene for activation of hepatic stellate cells, is regulated by Notch1 and TGF- $\beta$ /BMP signaling. World J Gastroenterol 21: 878-887, 2015.

16. Hu B, Wu Z, Bai D, Liu T, Ullenbruch MR and Phan SH: Mesenchymal deficiency of Notch1 attenuates bleomycin-induced pulmonary fibrosis. Am J Pathol 185: 3066-3075, 2015.

17. Xu Y, Liu W, Fang B, Gao S and Yan J: Artesunate ameliorates hepatic fibrosis induced by bovine serum albumin in rats through regulating matrix metalloproteinases. Eur J Pharmacol 744: 1-9, 2014.

18. Wang CM, Chen J, Jiang M, Xuan XP and Li HX: Relationship between artesunate influence on the process of TGF-betal induced alveolar epithelial cells transform into mesenchymal cells and on idiopathic pulmonary fibrosis. Yao Xue Xue Bao 49: 142-147, 2014 (In Chinese). 